\title{
Global Dynamics of a Higher Order Difference Equation with a Quadratic Term
}

\author{
Erkan Taşdemir \\ Kırklareli University, Pınarhisar Vocational School, Kırklareli, Turkey \\ erkantasdemir@hotmail.com
}

November 19, 2020

\begin{abstract}
In this paper, we investigate the dynamics of following higher order difference equation

$$
x_{n+1}=A+B \frac{x_{n}}{x_{n-m}^{2}}
$$

with $A, B$ and initial conditions are positive numbers, and $m \in\{2,3, \cdots\}$. Especially we study the boundedness, periodicity, semi-cycles, global asymptotically stability and rate of convergence of solutions of related higher order difference equations.

Keywords: Difference equations, periodicity, boundedness, semicycle, global asymptotic stability

AMS Subject Classification: 39A10, 39A23, 39A30

\section{Introduction}

Difference equations and their systems have captured the attention of the researchers over the last two decades. This attention result from area of usage of difference equations. In particular they which arise in mathematical models that describe problems in ecology, probability and engineering, etc. Since we know very little about such equations, it is very important to study higher order difference equations.

In [23], Jafar et al handled the following higher order rational difference equation$$
x_{n+1}=\frac{\beta x_{n}+\gamma x_{n-k}}{A+B x_{n}+C x_{n-k}}
$$

where the parameters $\beta, \gamma, A, B$ and $C$ and the initial conditions are nonnegative real numbers, $k=\{1,2, \ldots\}$. They studied the boundedness, invariant intervals, semi-cycles and global stability of related equation.
\end{abstract}


In [24], Saleh et al analyzed the invariant intervals, periodic character, the character of semi-cycles and global asymptotic stability of all positive solutions of difference equation

$$
x_{n+1}=\frac{\alpha+\beta x_{n}+\gamma x_{n-k}}{B x_{n}+C x_{n-k}}
$$

where the parameters $\alpha, \beta, \gamma, B$ and $C$ and the initial conditions are nonnegative real numbers, $k=\{1,2, \ldots\}$.

In [12], Devault et al investigated the boundedness, global stability and periodic character of solutions of the difference equation

$$
x_{n+1}=p+\frac{x_{n-k}}{x_{n}}
$$

where $p$ and the initial conditions are arbitrary positive numbers.

In [14], Saleh et al studied global asymptotic stability, periodicity and semicycle analysis of the unique positive equilibrium of following difference equation

$$
y_{n+1}=A+\frac{y_{n-k}}{y_{n}}
$$

where $A$ is positive and $k \in\{2,3, \cdots\}$.

In [8], Abu-Saris et al examined the global stability of unique positive equilibrium point of following higher order difference equations

$$
y_{n+1}=A+\frac{y_{n}}{y_{n-k}}
$$

where $A$ is positive and $k \in\{2,3, \cdots\}$. Additionally, in [17], Saleh et al dealt with the global asymptotic stability of the negative equilibrium of the difference equation (1) where $A<0$ and $k \in\{1,2, \cdots\}$.

In [11], Hassan dealt with dynamics of following difference equation

$$
x_{n+1}=p x_{n}+\frac{q}{x_{n-1}^{2}}
$$

where $p$ and $q$ lie in $(0,1)$.

In [10], Bešo et al investigated boundedness, global attractivity and NeimarkSacker bifurcation of following difference equation

$$
x_{n+1}=\gamma+\delta \frac{x_{n}}{x_{n-1}^{2}}
$$

where $\gamma, \delta$ are positive real numbers and the initial conditions are positive real numbers.

Motivated by the above studies, we consider the dynamics of following higher order difference equation

$$
x_{n+1}=A+B \frac{x_{n}}{x_{n-m}^{2}}
$$


where $A, B$ are positive real numbers, and $m \in\{2,3, \cdots\}$, and the initial conditions are positive numbers. Additionally, we investigate the boundedness, periodicity, global asymptotic stability and rate of convergence of related higher order difference equations.

Now, we present some important theorems which used by us during this study.

Theorem 1 (See [4]) Assume that $q_{i} \in \mathbb{R}, i=1,2, \cdots$, and $k \in\{0,1, \cdots\}$. Then

$$
\sum_{i=0}^{k}\left|q_{i}\right|<1
$$

is a sufficient condition for the asymptotic stability of the difference equation

$$
x_{n+k}+q_{1} x_{n+k-1}+\ldots+q_{k} x_{n}=0, n=0,1, \cdots .
$$

Theorem 2 ([13], p. 18) Let $f:[a, b]^{k} \rightarrow[a, b]$ be a continuous function, where $k$ is a positive integer, and where $[a, b]$ is an interval of real numbers and consider the following difference equation

$$
x_{n+1}=f\left(x_{n}, \cdots, x_{n-k}\right), n=0,1, \cdots .
$$

Suppose that $f$ satisfies the following conditions:

i. For each integer $i$ with $1 \leq i \leq k+1$, the function $f\left(z_{1}, z_{2}, \cdots, z_{k+1}\right)$ is weakly monotonic in $z_{i}$ for fixed $z_{1}, z_{2}, \cdots, z_{i-1}, z_{i+1}, \cdots, z_{k+1}$.

ii. If $(m, M)$ is a solution of the system

$$
m=f\left(m_{1}, m_{2}, \cdots, m_{k+1}\right) \text { and } M=f\left(M_{1}, M_{2}, \cdots, M_{k+1}\right),
$$

then $m=M$, where for each $i=1,2, \cdots, k+1$, we set

$$
m_{i}=\left\{\begin{array}{l}
m, \text { if } f \text { nondecreasing in } z_{i}, \\
M, \text { if } f \text { nonincreasing in } z_{i},
\end{array}\right\}
$$

and

$$
M_{i}=\left\{\begin{array}{l}
M, \text { if } f \text { nondecreasing in } z_{i}, \\
m, \text { if } f \text { nonincreasing in } z_{i} .
\end{array}\right\}
$$

Then there exists exactly one equilibrium point $\bar{x}$ of the difference equation (3), and every solution of (3) converges to $\bar{x}$.

Consider the scalar $k$ th-order linear difference equation

$$
x(n+k)+p_{1}(n) x(n+k-1)+\cdots+p_{k}(n) x(n)=0,
$$

where $k$ is a positive integer and $p_{i}: \mathbb{Z}^{+} \rightarrow \mathbb{C}$ for $i=1, \cdots, k$. Assume that

$$
q_{i}=\lim _{k \rightarrow \infty} p_{i}(n), i=1, \cdots, k,
$$

exist in $\mathbb{C}$. Consider the limiting equation of (4):

$$
x(n+k)+q_{1} x(n+k-1)+\cdots+q_{k} x(n)=0 .
$$


Theorem 3 (Poincaré's Theorem) Consider (4) subject to condition (5). Let $\lambda_{1}, \cdots, \lambda_{k}$ be the roots of the characteristic equation

$$
\lambda^{k}+q_{1} \lambda^{k-1}+\cdots+q_{k}=0
$$

of the limiting equation (6) and suppose that $\left|\lambda_{i}\right| \neq\left|\lambda_{j}\right|$ for $i \neq j$. If $x(n)$ is a solution of (4), then either $x(n)=0$ for all large $n$ or there exists an index $j \in\{1, \cdots, k\}$ such that

$$
\lim _{n \rightarrow \infty} \frac{x(n+1)}{x(n)}=\lambda_{j}
$$

The following results were obtained by Perron, and one of Perron's results was improved by Pituk, see [19].

Theorem 4 Suppose that (5) holds. If $x(n)$ is a solution of (4), then either $x(n)=0$ eventually or

$$
\lim _{n \rightarrow \infty} \sup \left(\left|x_{j}(n)\right|\right)^{1 / n}=\lambda_{j} .
$$

where $\lambda_{1}, \cdots, \lambda_{k}$ are the (not necessarily distinct) roots of the characteristic equation (7).

\section{Analysis of the periodicity, boundedness, semi- cycles, and global stability of solutions of Eq.(2)}

In this section, we firstly investigate the existence of two periodic solutions of Eq.(2) as $m$ is odd or even. We also study the boundedness and persistence of solutions of Eq.(2). Moreover we find out the semi-cycles of solutions of Eq.(2). Then we scrutiny the global asymptotic stability of solutions of Eq.(2). We further handle the rate of convergence of Eq.(2).

First of all, we take the change of the variables for Eq.(2) as follows $y_{n}=\frac{x_{n}}{A}$. From this, we obtain the following difference equation

$$
y_{n+1}=1+p \frac{y_{n}}{y_{n-m}^{2}}
$$

where $p=\frac{B}{A^{2}}$. From now on, we handle the difference equation (8). The unique positive equilibrium point of Eq.(8) is

$$
\bar{y}=\frac{1+\sqrt{1+4 p}}{2} .
$$

In this here, we study the periodic solutions of Eq.(8) with period two.

Theorem 5 Let $\left\{y_{n}\right\}$ be a positive solution of Eq.(8). Then Eq.(8) has no two periodic solution. 
Proof. We assume that there exist two periodic solution such that

$$
\cdots, \alpha, \beta, \alpha, \beta, \cdots
$$

where $\alpha$ and $\beta$ are positive and distinct real numbers. We handle two cases for the proof of Theorem. Firstly we consider a case such that $m$ is even. We have from Eq.(8)

$$
\alpha=1+\frac{p}{\beta}, \beta=1+\frac{p}{\alpha} .
$$

Hence we obtain that

$$
\alpha^{2}-\alpha-p=0
$$

So we get $\alpha=\frac{1+\sqrt{1+4 p}}{2}=\bar{y}=\beta$ which is a trivial solution. Now we deal with the other case such that $m$ is odd. Now we apply Elsayed's new method for two periodic solution, see [18]. We have from Eq.(8)

$$
\alpha=1+\frac{p \beta}{\alpha^{2}}, \beta=1+\frac{p \alpha}{\beta^{2}} .
$$

Hence if we take $\alpha=\beta n$ for $n \in \mathbb{R}-\{0,1,-1\}$. Therefore we obtain that

$$
\begin{aligned}
\beta n & =1+\frac{p}{n^{2} \beta}, \\
\beta & =1+\frac{p n}{\beta} .
\end{aligned}
$$

Thus, subtracting (10) from (9) gives the following

$$
\beta(n-1)=\frac{p}{\beta}\left(\frac{1}{n^{2}}-n\right)=\frac{p}{\beta} \frac{1-n^{3}}{n^{2}} .
$$

From $n \neq 1$, we have

$$
\begin{aligned}
\beta^{2} & =\frac{-p\left(n^{2}+n+1\right)}{n^{2}}, \\
\beta & = \pm \sqrt{\frac{-p\left(n^{2}+n+1\right)}{n^{2}}} .
\end{aligned}
$$

Since $\beta$ is real number, (11) is impossible for all real $n$ and $p>0$. This is a contradiction. So the proof is completed.

Now, we investigate the boundedness of solutions of Eq.(8).

Theorem 6 Every solutions of Eq.(8) is bounded and persist such that

$$
1<y_{n}<1+p(1+p)^{m}
$$

for $n \geq 2 m+2$. 
Proof. Let $\left\{y_{n}\right\}$ be a positive solution of Eq.(8) and $p>0$. Then, we have from Eq.(8)

$$
\begin{aligned}
& y_{1}=1+p \frac{y_{0}}{y_{-m}^{2}}>1, \\
& y_{2}=1+p \frac{y_{1}}{y_{1-m}^{2}}>1 .
\end{aligned}
$$

Thus we obtain by induction $y_{n}>1$ for all $n \geq 1$.

Now we consider the other side. We have from Eq.(8)

$$
y_{n}=1+p \frac{y_{n-1}}{y_{n-m-1}^{2}}=1+\frac{p}{y_{n-m-1}}\left(\frac{y_{n-1}}{y_{n-2}} \frac{y_{n-2}}{y_{n-3}} \cdots \frac{y_{n-m}}{y_{n-m-1}}\right) .
$$

We know that $y_{n}>1$ for all $n \geq 1$. So we obtain from (12),

$$
y_{n}<1+p\left(\frac{y_{n-1}}{y_{n-2}} \frac{y_{n-2}}{y_{n-3}} \cdots \frac{y_{n-m}}{y_{n-m-1}}\right) .
$$

Additinally, we set Eq.(8) such that

$$
y_{i}=1+p \frac{y_{i-1}}{y_{i-m-1}^{2}} \Rightarrow \frac{y_{i}}{y_{i-1}}=\frac{1}{y_{i-1}}+\frac{p}{y_{i-m-1}^{2}}
$$

for $i=1,2, \cdots$. Thus we have from (13) and (14)

$y_{n}<1+p\left(\frac{1}{y_{n-2}}+\frac{p}{y_{n-m-2}^{2}}\right)\left(\frac{1}{y_{n-3}}+\frac{p}{y_{n-m-3}^{2}}\right) \cdots\left(\frac{1}{y_{n-m-1}}+\frac{p}{y_{n-2 m-1}^{2}}\right)$.

If $n \geq 2 m+2$, we have $\left(y_{i}\right)_{i=2}^{2 m+1}>1$. So, we get

$$
y_{n}<1+p(1+p)^{m}
$$

for $n \geq 2 m+2$.

Now we deal with the semi-cycle analysis of solutions of Eq.(8). The following theorem gives some valuable results about oscillation behaviours of solutions of Eq.(8).

Theorem 7 Every solution of Eq.(8) satisfies following statements:

(a) Every solution of Eq.(8) has semi-cycles of length at most $2 m+1$.

(b) If every solution of Eq.(8) has a semi-cycle of length at least $k$, then the following semi-cycle has at least $k+1$ terms.

Proof. Let $\left\{y_{n}\right\}$ be a solution of Eq.(8). We firstly consider a negative semicycle. The positive semi-cycle is similar and can be omitted. Suppose that a solution $\left\{y_{n}\right\}$ of Eq.(8) has a negative semi-cycle with $2 m+1$ terms. Assume that $y_{N}$ is the first term in this negative semi-cycle. Thus we have

$$
y_{N}, y_{N+1}, \cdots, y_{N+2 m}<\bar{y} \text {. }
$$


Additionally, we get the followings for $i=1,2, \cdots, m$

$y_{N+m+i}=1+p \frac{y_{N+m+i-1}}{y_{N+i-1}^{2}}>1+p \frac{y_{N+m+i-1}}{\bar{y}^{2}}>y_{N+m+i-1}\left(1+\frac{p}{\bar{y}^{2}}\right)>y_{N+m+i-1}$.

Hence we obtain that

$$
y_{N+m}<y_{N+m+1}<\cdots<y_{N+2 m-1}<y_{N+2 m} .
$$

Therefore we get $y_{N+m}<y_{N+2 m}$. So we have the followings

$$
y_{N+2 m+1}=1+p \frac{y_{N+2 m}}{y_{N+m}^{2}}>\bar{y} .
$$

Now we handle the proof of (b). Assume that Eq.(8) has a semi-cycle of length at least $k$. We again consider a negative semi-cycle. The positive semicycle is similar. Let $y_{N}$ be the first term of the following positive semi-cycle. Hence

$$
y_{N-m}, y_{N-m+1}, \cdots, y_{N-1}<\bar{y}<y_{N} .
$$

Therefore we obtain that

$$
y_{N+1+i}=1+p \frac{y_{N+i}}{y_{N-m+i}^{2}}>\bar{y}
$$

for $i=0,1, \cdots, k-1$. Thus negative semi-cycle occurs at least $m+1$ terms. So, every semi-cycle of following this semi-cycle consist at least $m+1$ terms.

In this here, we study the stability of Eq.(8). Firstly we handle the linearized equation of Eq.(8) about its unique positive equilibrium point. Let $I$ be some interval of real numbers and let

$$
f: I^{m+1} \rightarrow I
$$

be a continuously differentiable function such that $f$ is defined by

$$
f\left(y_{n}, y_{n-1}, \cdots, y_{n-m}\right)=1+p \frac{y_{n}}{y_{n-m}^{2}} .
$$

Therefore we have

$$
\begin{gathered}
q_{0}=\frac{\partial f}{\partial y_{n}}=\frac{p}{\bar{y}^{2}} \\
q_{1}=q_{2}=\cdots=q_{m-1}=0 \\
q_{m}=\frac{\partial f}{\partial y_{n-m}}=-\frac{2 p}{\bar{y}^{2}} .
\end{gathered}
$$

Hence we obtain the linearized equation of Eq.(8) about its unique positive equilibrium point $\bar{y}$ as follow:

$$
z_{n+1}-\frac{p}{\bar{y}^{2}} z_{n}+\frac{2 p}{\bar{y}^{2}} z_{n-m}=0
$$


Therefore, the characteristic equation of Eq.(8) is

$$
\lambda^{m+1}-\frac{p}{\bar{y}^{2}} \lambda^{m}+\frac{2 p}{\bar{y}^{2}}=0 .
$$

Theorem 8 Let $p \in\left(0, \frac{3}{4}\right)$. Then the equilibrium point $\bar{x}$ of Eq.(8) is locally asymptotically stable.

Proof. From (15), we have

$$
\left|q_{1}\right|+\left|q_{2}\right|+\cdots\left|q_{m}\right|=\frac{3 p}{\bar{y}^{2}} .
$$

Note that

$$
\frac{p}{\bar{y}^{2}}=\frac{2 p+1-\sqrt{4 p+1}}{2 p}
$$

Thus

$$
\begin{aligned}
\left|q_{1}\right|+\left|q_{2}\right|+\cdots\left|q_{m}\right| & =\frac{3 p}{\bar{y}^{2}}<1, \\
\frac{3(2 p+1-\sqrt{4 p+1})}{2 p} & <1, \\
\frac{4 p+3-3 \sqrt{4 p+1}}{2 p} & <0 .
\end{aligned}
$$

Hence, we get from $p>0$,

$$
(\sqrt{4 p+1}-1)(\sqrt{4 p+1}-2)<0
$$

So, we obtain $0<p<\frac{3}{4}$. Therefore, the proof of Theorem 8 is completed.

Theorem 9 Let $0<p<\frac{3}{4}$. Then the equilibrium point $\bar{y}$ of Eq.(8) is globally asymptotically stable.

Proof. Firstly, we consider the following function

$$
f(u, v)=f\left(y_{n}, y_{n-m}\right)=1+p \frac{y_{n}}{y_{n-m}^{2}},
$$

The function $f(u, v)$ is nondecreasing in $u$ and nonincreasing in $v$. Let $(m, M)$ is a solution of the system

$$
m=f(m, M) \text { and } M=f(M, m) .
$$

Hence we obtain that

$$
m=1+p \frac{m}{M^{2}}, M=1+p \frac{M}{m^{2}} .
$$

Therefore we have $m=M$. According to Theorem 2, every solution of Eq.(8) converges to $\bar{x}$, as desired.

Here, we handle the rate of convergence of Eq.(8). 
Theorem 10 Every solution of Eq.(8) satisfies both of the following asymptotic relations

$$
\begin{aligned}
\lim _{n \rightarrow \infty}\left|\frac{y_{n+1}-\bar{y}}{y_{n}-\bar{y}}\right| & =\left|\lambda_{j}\right|, \\
\lim _{n \rightarrow \infty} \sup \left(\left|y_{n}-\bar{y}\right|\right)^{1 / n} & =\left|\lambda_{j}\right|
\end{aligned}
$$

where $j \in\{1, \cdots, k\}$ and $\lambda_{j}$ are the roots of characteristic equation (16).

Proof. We get from Eq.(8):

$$
\begin{aligned}
y_{n+1}-\bar{y} & =\left(1+p \frac{y_{n}}{y_{n-m}^{2}}\right)-\left(1+p \frac{\bar{y}}{\bar{y}^{2}}\right) \\
& =\frac{p}{y_{n-m}^{2}}\left(y_{n}-\bar{y}\right)-\frac{p\left(y+y_{n-m}\right)}{\bar{y} \cdot y_{n-m}^{2}}\left(y_{n-m}-\bar{y}\right) .
\end{aligned}
$$

Set $e_{n}=y_{n}-\bar{y}$. Therefore we have

$$
e_{n+1}+p_{n} e_{n}+q_{n} e_{n-m}=0
$$

where

$$
p_{n}=-\frac{p}{y_{n-m}^{2}}, q_{n}=\frac{p\left(y+y_{n-m}\right)}{\bar{y} \cdot y_{n-m}^{2}} .
$$

Due to the equilibrium point $\bar{y}$ of Eq.(8) is globally asymptotically stable, we get

$$
\lim _{n \rightarrow \infty} p_{n}=-\frac{p}{\bar{y}^{2}}, \lim _{n \rightarrow \infty} q_{n}=\frac{2 p}{\bar{y}^{2}} .
$$

Hence, the limiting equation of Eq.(8) is the linearized equation (15).

\section{Numerical Simulations of Eq.(2)}

This section, we present two numerical examples for verify our theoretical results.

Example 11 Consider Eq.(2) for $A=\sqrt{10}, B=7$, and $m=5$. Then we have following difference equation

$$
x_{n+1}=\sqrt{10}+7 \frac{x_{n}}{x_{n-5}^{2}} .
$$

Now, we consider the change of the variables for Eq.(17) as follows $y_{n}=\frac{x_{n}}{\sqrt{10}}$. Thus we obtain that $p=\frac{B}{A^{2}}=0.7$, and we get the following sixth order difference equation

$$
y_{n+1}=1+0.7 \frac{y_{n}}{y_{n-5}^{2}}
$$

Let the initial conditions are $y_{-5}=10, y_{-4}=6, y_{-3}=8, y_{-2}=4, y_{-1}=3$ and $y_{0}=5$. Then unique positive equilibrium point $\bar{y}=1.4747$ of Eq.(18) is globally asymptotically stable. Figure 1 shows the first 600 terms of Eq.(18). 


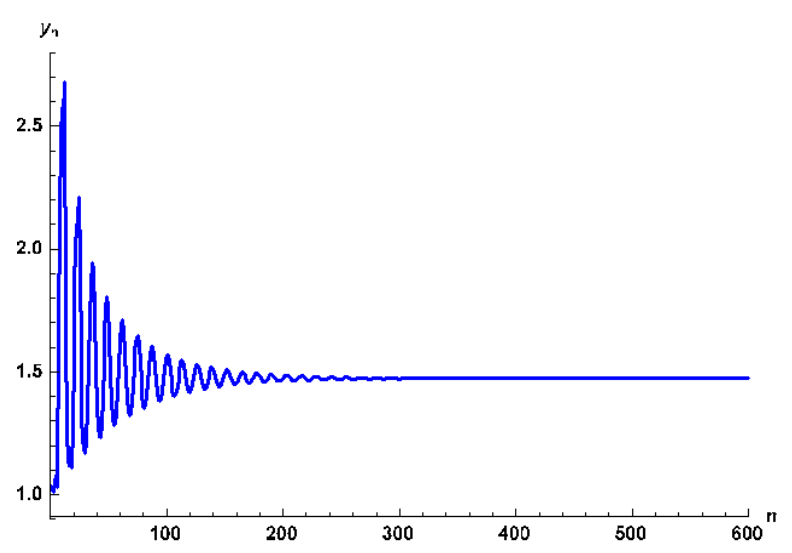

Figure 1: Plot of Eq.(18).

Example 12 Consider Eq.(2) for $A=3, B=18$, and $m=4$. Then we have following difference equation

$$
x_{n+1}=3+18 \frac{x_{n}}{x_{n-4}^{2}} .
$$

Now, we consider the change of the variables for Eq.(19) as follows $y_{n}=\frac{x_{n}}{3}$. Thus we obtain that $p=\frac{B}{A^{2}}=2$, and we get the following fifth order difference equation

$$
y_{n+1}=1+2 \frac{y_{n}}{y_{n-4}^{2}} .
$$

Let the initial conditions are $y_{-4}=1.6, y_{-3}=0.8, y_{-2}=1, y_{-1}=0.3$ and $y_{0}=$ 2. Then every solution of Eq.(20) oscillate about unique positive equilibrium point $\bar{y}=2$. Figure 2 shows the first 1000 terms of Eq.(20).

\section{Conclusion and Open Problems}

During this paper, we investigate the dynamics of difference equation (8). We firstly find out that Eq.(8) has not periodic solution with period two. Then we reveal the bounded solution of Eq.(8). We further study the semi-cycles of Eq.(8). Moreover, we discover that the equilibrium point $\bar{y}$ of Eq.(8) is globally asymptotically stable. Additionally, we study the rate of convergence of Eq.(8). Finally we present two numerical examples in order to verify our theoretical results.

Open Problem 1: Investigate the dynamics of following higher order difference equation

$$
x_{n+1}=A+B \frac{x_{n}}{x_{n-m}^{r}}
$$

where the initial values are real numbers and $r \in\{3,4, \cdots\}$. 


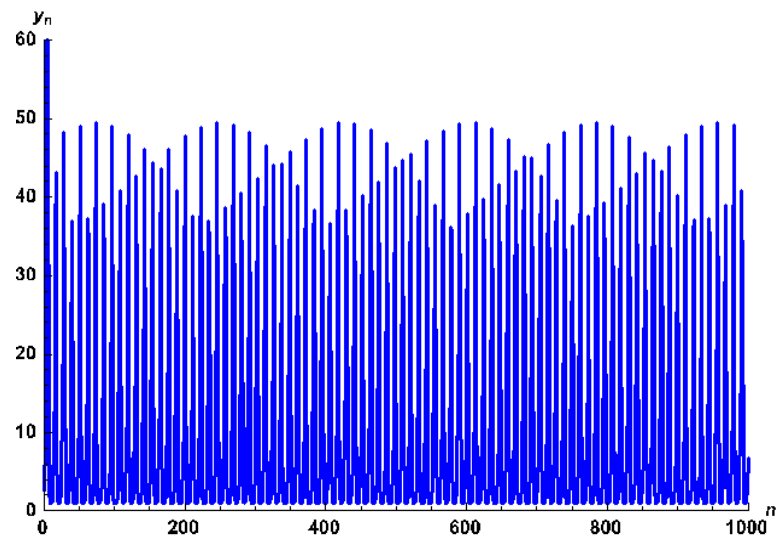

Figure 2: Plot of Eq.(20).

Open Problem 2: Investigate the dynamics of following higher order difference equation

$$
x_{n+1}=A+B \frac{x_{n}^{q}}{x_{n-m}^{r}}
$$

where the initial values are real numbers and $r \in\{2,3, \cdots\}, q \in\{2,3, \cdots\}$.

\section{References}

[1] S. Elaydi, An Introduction to Difference Equations, Springer-Verlag, New York, 1996.

[2] M.R.S. Kulenovic, G. Ladas, Dynamics of Second Order Rational Difference Equations with Open Problems and Conjectures, Chapman \& Hall/CRC, Boca Raton, 2002

[3] E. Camouzis, G. Ladas, Dynamics of third order rational difference equations with open problems and conjectures, volume 5 of Advances in Discrete Mathematics and Applications, Chapman \& Hall/CRC, Boca Raton, 2008.

[4] V.L. Kocic, G. Ladas, Global Asymptotic Behavior of Nonlinear Difference Equations of Higher Order with Applications, Kluwer Academic Publishers, Dordrecht, 1993.

[5] S.A. Kuruklis, The asymptotic stability of $x n+1$ axn + bxn $k=0$, J. Math. Anal. Appl. 188 (1994) 719-731.

[6] V.G. Papanicolaou, On the asymptotic stability of a class of linear difference equations, Math. Mag. 69 (1996) 34-43.

[7] D. Şimşek, B. Oğul, C. Çınar, Solution of the rational difference equation $\mathrm{xn}+1=\mathrm{xn}-17 / 1+\mathrm{xn}-5 \mathrm{xn}-11$, Filomat 33(5) (2019) 1353-1359. 
[8] R. Abu-Saris, R. DeVault, Global stability of yn+ 1= A+ yn/yn- k, Appl. Math. Lett. 16 (2003) 173-178.

[9] A. Bilgin and M.R.S. Kulenovic, Global asymptotic stability for discrete single species population models, Discrete Dyn. Nat. Soc., 2017 (2017) 115 .

[10] E. Bešo, S. Kalabušić, N. Mujić, E. Pilav, Boundedness of solutions and stability of certain second-order difference equation with quadratic term. Adv. Differ. Equ. 2020(19) (2020) 1-22. https://doi.org/10.1186/s13662019-2490-9

[11] S.S. Hassan, Dynamics of the Rational Difference Equation $\quad \mathrm{xn}+1=\mathrm{pxn}+\mathrm{qx} 2 \mathrm{n}-1 . \quad$ Preprints 2020, 2020040113 (doi: 10.20944/preprints202004.0113.v1).

[12] R. DeVault, C. Kent, W. Kosmala, On the recursive sequence $\mathrm{xn}+1=\mathrm{p}+(\mathrm{xn}-\mathrm{k} / \mathrm{xn})$, J. Difference Equ. Appl., 9 (8) (2003) 721-730.

[13] E.A. Grove, G. Ladas, Periodicities in Nonlinear Difference Equations, Vol. 4, Chapman \& Hall / CRC, 2005.

[14] M. Saleh, M. Aloqeili, On the rational difference equation $y n+1=\mathrm{A}+$ yn-k yn, Appl. Math. Comput. 171(1) (2005) 862-869.

[15] M. Saleh, S. Abu-Baha, Dynamics of a higher order rational difference equation, Appl. Math. Comput. 181 (2006) 84-102.

[16] M. Göcen, A. Cebeci, On the periodic solutions of some systems of higher order difference equations. Rocky Mt. J. Math. 48(3) (2018) 845-858.

[17] M. Saleh, M. Aloqeili, On the difference equation $\mathrm{yn}+1=\mathrm{A}+\mathrm{yn} / \mathrm{yn}-\mathrm{k}$ with $\mathrm{A}<0$. Appl. Math. Comput. 176(1) (2006) 359-363.

[18] E.M. Elsayed, New method to obtain periodic solutions of period two and three of a rational difference equation, Nonlinear Dyn., 79 (2015) 241-250.

[19] M. Pituk, More on Poincaré's and Perron's theorems for difference equations, J. Difference Equ. Appl. 8(3) (2002) 201-216.

[20] İ. Okumuş, Y. Soykan, Dynamical behavior of a system of three-dimensional nonlinear difference equations, Adv. Differ. Equ. 2018:224 (2018) 1-15.

[21] Y.H. Su, W.T. Li, Global attractivity of a higher order nonlinear difference equation, J. Difference Equ. Appl. 11(10) (2005) 947-958.

[22] E.M.E. Zayed, M.A. El-Moneam, On the rational recursive two sequences $\mathrm{xn}+1=$ axn-k+bxn-k/(cxn+dxn-k), Acta Math. Vietnamica, 35 (2010) 355369. 
[23] A. Jafar, M. Saleh, Dynamics of nonlinear difference equation $\mathrm{xn}+1=\beta \mathrm{xn}+\gamma \mathrm{xn}-\mathrm{kA}+\mathrm{Bxn}+\mathrm{Cxn}-\mathrm{k}$, J. Appl. Math. Comput. 57, (2018) 493-522.

[24] M. Saleh, N. Alkoumi, A. Farhat, On the dynamics of a rational difference equation $\mathrm{xn}+1=\alpha+\beta \mathrm{xn}+\gamma \mathrm{xn}-\mathrm{kBxn}+\mathrm{Cxn}-\mathrm{k}$, Chaos Solitons Fractals, 96 (2017) 76-84. 